\title{
Nasal Vestibule Squamous Cell Papilloma
}

National Cancer Institute

\section{Source}

National Cancer Institute. Nasal Vestibule Squamous Cell Papilloma. NCI Thesaurus.

Code C4369.

A benign exophytic squamous cell neoplasm with papillary growth that arises from the nasal vestibule. 